\title{
Modeling of Photonics Alcohol Detector System Considering Syari'ah Compliance Beverages
}

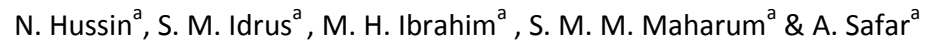 \\ ${ }^{a}$ Faculty of Electrical Engineering, Universiti Teknologi Malaysia, 81310 UTM Johor Bahru \\ *Corresponding author: beehah_phoenix@yahoo.com
}

\section{Article history}

Received: 8 March 2012

Received in revised form: 10 April

2012

Accepted: 18 July 2012

Graphical abstract

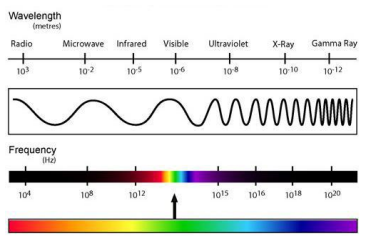

\section{Abstract}

The remarkable growth in the Halal sector has initiated a growing interest among researchers in developing new, rapid and reliable methods for Halal verification. The need for proper supervision, certification and labelling should follow closely to offer reliable Halal drinks products. The percentage of ethanol in the beverages is directly determined by using the first derivative of near infrared (NIR) absorbance spectrum where the range of wavelength used is from $1680 \mathrm{~nm}$ to $1700 \mathrm{~nm}$. Therefore a new method of alcohol detection for beverage had been studied in the reason of helping the consumers especially the Muslims in identifying whether the beverage that they intend to purchase is Halal or Haram, and later the alcohol detector system that is low cost and reliable in detecting the presence of alcohol in our drinks can be modelled. Finally, by making suitable assumptions of the NIR technique, the alcohol detector system was designed, modelled and simulated by using Microsoft Excel and Microsoft Visual Basic.

Keywords: Alcohol; NIRS; ethanol; beverages; Microsoft Visual Basic; halal

C 2012 Penerbit UTM Press. All rights reserved.

\subsection{INTRODUCTION}

Alcoholic drinks or beverages are totally prohibited in Islam, and even a small amount of the drink added into foods or drinks will render the products haram. However, trace amounts of ethanol (naturally present or ethanol used in food processing) is allowed if the amount is insufficient to cause intoxication. The food industry is a complex, global collective of diverse businesses that together supply much of the food energy consumed by the world population. But are we aware of the contents inside it? Halal sign is of paramount importance to ensure food and beverage in takes is legitimate in religion.

In chemistry, an alcohol is any organic compound in which a hydroxyl functional group (-OH) is bound to a carbon atom, usually connected to other carbon or hydrogen atoms. Ethanol $\left(\mathrm{C}_{2} \mathrm{H}_{5} \mathrm{OH}\right)$ is the type of alcohol found in alcoholic beverages, and in common speech the word alcohol refers specifically to ethanol. Alcohol, generally on the other hand refers to the genuine ethanol and ethanol denature in any density. Alcohol can be obtained from various sources, either from natural process named fermentation, chemical reaction of petrol or from rotting process of organic compound. Considering the natural process, alcohol is produced through alcoholic fermentation of material containing sugar or starch. The overall process of fermentation is the conversion of glucose sugar $\left(\mathrm{C}_{6} \mathrm{H}_{12} \mathrm{O}_{6}\right)$ to alcohol $\left(\mathrm{CH}_{3} \mathrm{CH}_{2} \mathrm{OH}\right)$ and carbon dioxide gas $\left(\mathrm{CO}_{2}\right)$. As a reminder, the sugars used can be a range of fermentable sugars and these sugars are converted to glucose which is then converted to alcohol and carbon dioxide by certain enzymes. The ethanol produced from the fermentation process happens naturally and cannot be avoided.

Food ingredients containing alcohol can be divided into three parts [1]. The first part, fruit juice, milk, yogurt, and fermented dough contain a very small rate of alcohol which is not more than $0.5 \%$ but grape juice may contain $1 \%$ of alcohol. Second part is the food ingredients that contain a very small rate of alcohol. Usually alcohol is used to melt some of the material that does not melt in water, such as dyes, preservatives, flavours, and others. Finally the third part is the food and drinks that are mixed with the intoxicating liquor such as brandy to get a sense of unique, different and preferred by some people. In principle, alcohol may be presented in all types of juices especially from which one that could produce wine. Thus, as the grapes contain are derived from the natural yeasts that convert the sugar into the remnants of alcohol, grapes are naturally high on the list of juices that contain alcohol.

Near infrared (NIR) spectroscopy corresponds to a wavelength range of 3 between 700 and $2500 \mathrm{~nm}$ on the visible light side of the divided infrared spectrum (Figure 1). Infrared (IR) spectroscopy is the most spectroscopic techniques used by organic and inorganic chemists as the portion of the IR region is most useful for analysis of organic compounds [2]. NIRS can be used for non-invasive or non-destructive for internal quality measurement. Near infrared spectroscopy (NIRS) instruments are usually versatile for applying on different types of sample. NIRS is a rapid, precise, and non-destructive technique which can be well utilized in determination of sample quality. A basic NIRS 
measurement system consists of three parts, which are, a light source, a wavelength isolator, and a detector. Initially, light source will emit a NIR light toward the sample. NIR spectroscopy is widely used for both qualitative and quantitative determination of a variety of parameters. Near-infrared spectroscopy is based on molecular overtone and combination vibrations. Such transitions are forbidden by the selection rules of quantum mechanics. As a result, the molar absorptivity in the near IR region is typically quite small. One advantage is that NIR can typically penetrate much farther into a sample than mid infrared radiation.

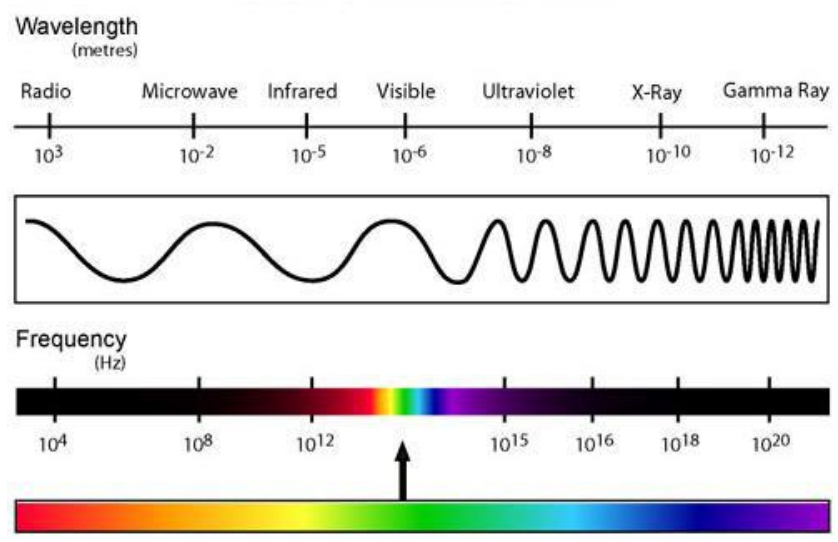

Figure 1 The electromagnetic spectrum highlighting the relationship between increased frequency and decreased frequency. The region of the spectrum we perceive as visible light corresponds to the range of $10^{-3} \mathrm{~m}$ (short wavelength edge of the microwave band) and $10^{-3} \mathrm{~m}$ (long wavelength edge of the ultraviolet band). Individual substances, molecular groups, molecules and atoms absorb or emit energy at characteristics regions of this spectrum

\subsection{SYSTEM DESIGN}

The main goal of IR spectroscopic analysis is to determine the chemical functional groups in the sample. Simply, it is the absorption measurement of different IR frequencies by a sample positioned in the path of an IR beam. Different functional groups will absorb different characteristic frequencies of IR radiation. Using various sampling accessories, IR spectrometers can accept a wide range of sample types such as gases, liquids, and solids. Therefore, IR spectroscopy is an important and popular tool for structural elucidation and compound identification. Figure 2 shows the block diagram of the system design.

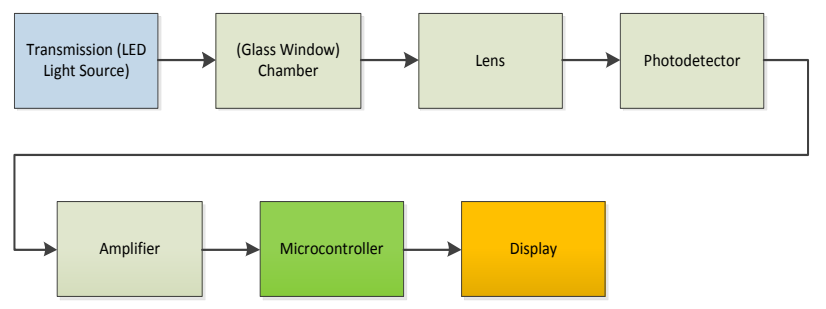

Figure 2 The block diagram of the system design

\subsection{Optical Source Light Emitting Diode}

IR absorption positions are generally presented as either wavenumbers $(v)$ or wavelengths $(\lambda)$. The wavenumber defines the number of waves per unit length and it is directly proportional to frequency.

While in optical system, light rays generated by optical sources carry the information. The common sources are the laser diodes (coherent source) and light-emitting diodes (incoherent source). The light-emitting diode (LED) is said to be incoherent source [3]. If the energy supplied is about the same as the gap energy $W_{g}$, free electrons and free holes will have sufficient energy to move into the junction region. In short, LED emits radiation through recombination of holes and electrons that are injected into the junction via forward bias voltage.

Referring to [4], it was found that the light source must be operated at a wavelength of $1680 \mathrm{~nm}$ where the ethanol absorbance is dominant at that particular wavelength. From the research, it was found that the Indium gallium arsenide (InGaAs) infrared LED L10823-01 series manufactured by Hamamatsu operates at wavelength range from $1600 \mathrm{~nm}$ to $1700 \mathrm{~nm}$. Clearly the intended operating wavelength of $1680 \mathrm{~nm}$ is included within the wavelength range. Infrared LED with different materials and alloys have different bandgap energies. InGaAs type was chosen since the required bandgap energy is $0.738 \mathrm{eV}$. The light source is consistently supplied with $50 \mathrm{~mA}$ and thus the power emitted by the infrared LED is $2.3 \mathrm{~mW}$.

\subsection{Glass Window (Chamber) \& Lens}

In the chamber the sample is inserted for the process of determining the ethanol concentration. This chamber is made from glass and is not required for the short pathlength. The flow cell is designed to be removal so that it easier to clean up before or after adding others sample. Lens was used to focus the light onto the optical detector. For the purpose of design, a photodetector was located at $3 \mathrm{~cm}$ from the lens. By applying the formula of maximum acceptance angle, the assumption of coupling efficiency can be made. Lens is very useful to improve the coupling efficiency of light onto the detector by $96 \%$ since the infrared LED radiates over large angle.

\subsection{Photodetector, Amplifier \& Microcontroller}

In this design, commercial light detector, InGaAs PIN Photodiode G8376-05 series manufactured by Hamamatsu in developing the system model has been proposed. The incident photon to the photodiode can be detected as the energy gap of $0.738 \mathrm{eV}$ is in between the acceptance gap energies of the photodiode (from $0.729 \mathrm{eV}$ up to $1.378 \mathrm{eV}$ ). According to [5], the absorption coefficient of a detector, $\alpha$ depends on the wavelength for different semiconductors used in the photodetector. Therefore, the value of $\alpha$ for InGaAs photodetector at $1680 \mathrm{~nm}$ is $2500 \mathrm{~cm}^{-1}$ and its depletion region, $d$ is $50 \mu \mathrm{m}$. An amplifier was used to amplify the voltage reading across the load resistance of $50 \Omega$ since the value was too small to be inserted into the microcontroller for the result processing. The maximum voltage reading can be accepted by the microcontroller is only $5 \mathrm{~V}$.

Different sample may result different voltage reading by the photodetector. Therefore, a microcontroller was suggested in this system design to process and differentiate that voltage reading which was assigned to different ethanol concentration. Since all the values of voltage reading according to specific ethanol 
concentration would be stored inside the microcontroller, a simple programming is required to compare the storage data with the voltage reading detected by the system. When the voltage reading matches one of the storage data, result of alcohol contain in the sample would be sent to the LCD for the purpose of result display.
Briefly, the system design consists of a flow cell that is made from glass, a light source and detector, an amplifier, and a microcontroller. The details of the system model can be found in the Figure 3.

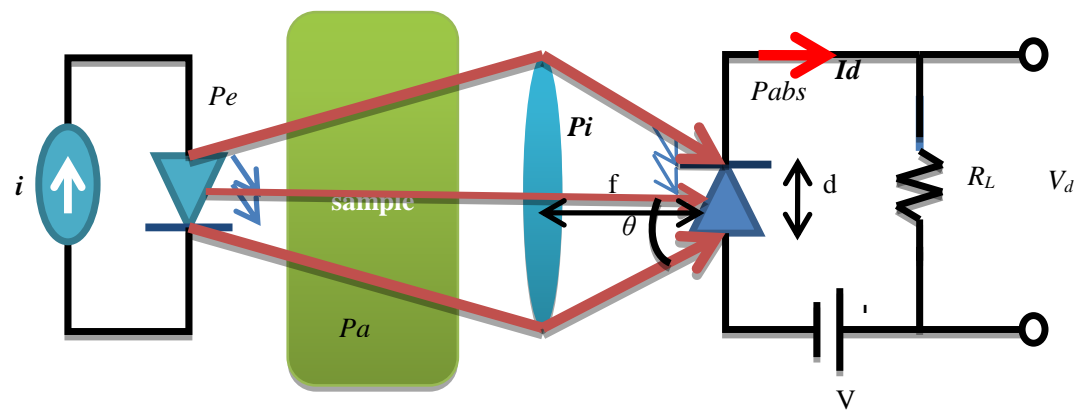

Figure 3 The system model

\subsection{SIMULATION RESULT}

The basis of most quantitative spectroscopic analyses is Beer's law. This law relates the amount of light absorbed by a sample, a spectroscopic observable property, to the concentration of absorbing species in the sample. This relationship is what allows absorbance measurements to be used to predict concentrations. Through the calibration line obtained as shown in Figure 4, the equation of $d A / d \lambda=0.0127 c$ where $c$ is the concentration of ethanol in $\% \mathrm{v} / \mathrm{v}$.

Two graphs were obtained from the calculation of the mathematical model from Figure 3 From the system model two graph were obtained which is the first graph (Figure 5) is power absorbed $P_{a}$ versus ethanol concentration while the second graph (Figure 6) show the current $\left(I_{d}\right)$ across the ethanol concentration. Figure 5 shows that the increasing of ethanol concentration will give influence to power absorbed. It means higher concentration will give greater $P_{a b s}$. While, Figure 6 show that the current is inversely proportional to the ethanol concentration. The ethanol concentration in a sample increase, more power radiated by the infrared LED, $P_{e}$ would be absorbed by the sample and thus the incident power to the photodetector, $P_{\mathrm{i}}$ would decrease along the increase of power absorbed by the sample, $P_{a}$. Therefore, the smaller the value of $P_{i}$ the smaller the successful power absorbed by the photodiode, $P_{a b s}$. Hence, the total current produced by the photodetector, $I_{d}$ would be smaller too.

\subsection{CONCLUSION}

By utilizing this novel method, it was found that the photonic alcohol detector system is possible to be a practical for prototype development in helping Muslim consumers, which cover up to $52 \%$ from $25,715,819$ of the total population, to identify whether the beverages they consumed is Halal or not. Besides that, the development of this system is applicable to be used by the alcohol allergy sufferers to alert them when the beverages contain higher percentage of alcohol. Even though, the system only shows a basic component of optical system but the system is able to detect the possible presence of ethanol in liquid and also it proven that light rays at specific wavelength $1680 \mathrm{~nm}$ are able to detect the presence of ethanol in liquid. The design and the model of alcohol detector was predicted to be a low cost system since the components that are used in this system are inexpensive such as window glass cell, infrared LED, PIN photodiode, amplifier, a microcontroller and the LCD screen.

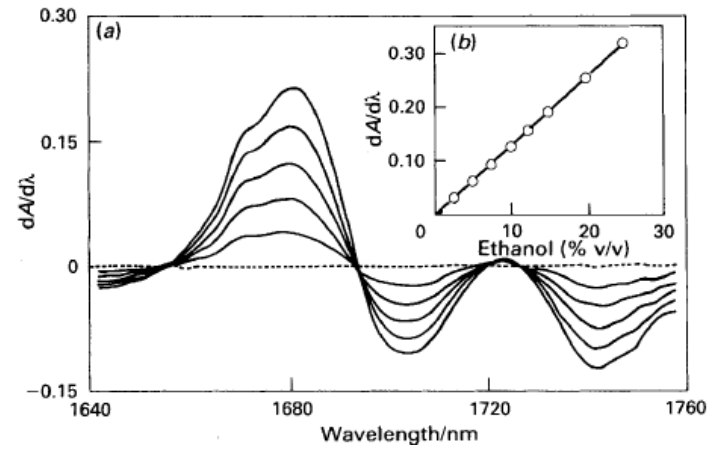

Figure 4 The calibration graph obtained from ethanol determination

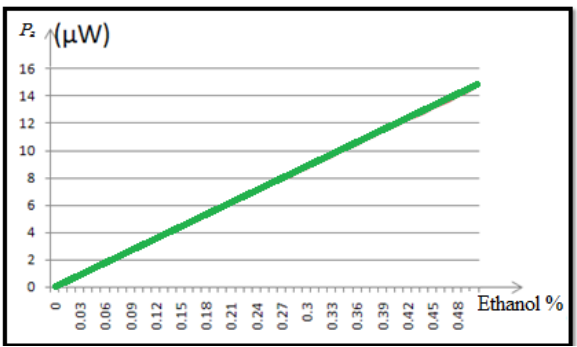

Figure $5 P_{2}$ versus concentration of ethanol

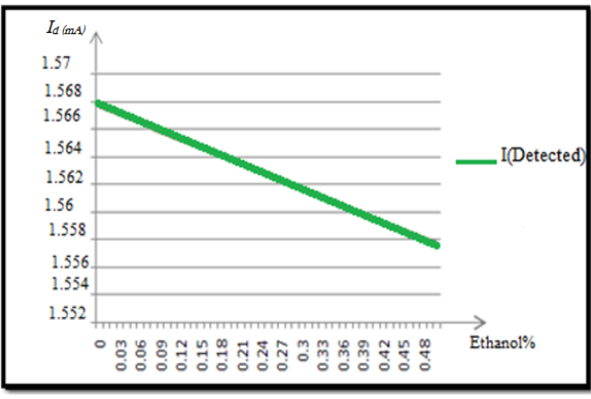

Figure 6 Current photodiode versus concentration of ethanol 


\section{References}

[1] A. K. Dutta. 1999. Optical devices for premises networks. SPIE Proceed. 3805. 194-208.

[2] C.-P. Sherman Hsu. Infrared Spectroscopy. Handbook of Instrumental Techniques for Analytical Chemistry. 249.

[3] John M. Senior. 1985. Optical fiber communications. Englewood Cliffs, N.J: Prentice Hall.

[4] Maximo Gallignani, Salvador Garrigues, and Miguel de la Guardia. 1993. Direct Determination of Ethanol in all Types of Alcoholic Beverages by Near-infrared Derivative Spectrometry. Analyst. 118: 1167-1173.

[5] A. K. Dutta, and M. Saif Islam. 2005. Novel Broadband Photodetector for Optical Communication. Proc. Of SPIE. 6014.
[5] A. K. Dutta, M. Takechi, R. S. Virk, M. Kobayashi, K. Araki, K. Sato, M. Gentrup, and R. Ragle, "40 Gb/s Postamplifier and PIN/preamplifier Receiver Moduled for Next Generation Optical Frontend System", IEEE J. Lightwave Technology, 20, pp. 2229-2238, 2002.

[6] T. Mikawa, S. Kagawa, and T. Kaneda, "InP/InGaAs PIN photodiodes in the $1 \mu \mathrm{m}$ wavelength regio." Fujitsu Sci. Tech. J., 20, pp. 201-218, 1984.

Shmidt Handbook Series on Semiconductor Parameters, vol. 1, M. Levinshtein, S. Rumyantsev and M. Shur, ed., World Scientific, London, pp. 169-190, 1996.

[7] Roman Benes, "Method and Device for Determining an Alcohol Content of Liquids", U.S. Patent 733A. Feb. 18, 2008. 